\title{
An Effective Image Contrast Enhancement Method Using Global Histogram Equalization
}

\author{
M. A. Yousuf ${ }^{*}$, and M. R. H. Rakib \\ Department of Computer Science and Engineering, Mawlana Bhashani Science and Technology \\ University, Santosh, Tangail 1902, Bangladesh
}

Received 17 June 2010, accepted in revised form 3 November 2010

\begin{abstract}
Image enhancement is one of the most important issues in low-level image processing. Histograms are the basis for numerous spatial domain processing techniques. In this paper, we present a simple and effective method for image contrast enhancement based on global histogram equalization. In this method, at first input image is normalized by making the minimum gray level value to 0 . Then the probability of each grey level is calculated from the available ROI grey levels. Finally, histogram equalization is performed on the input image based on the calculated probability density (or distribution) function. As a result, the mean brightness of the input image does not change significantly by the histogram equalization. Additionally, noise is prevented from being greatly amplified. Experimental results on medical images demonstrate that the proposed method can enhance the images effectively. The result is also compared with the result of image enhancement technique using local statistics.
\end{abstract}

Keywords: Histogram equalization; Global histogram equalization; Image enhancement; Local statistics.

(c) 2011 JSR Publications. ISSN: 2070-0237 (Print); 2070-0245 (Online). All rights reserved.

doi:10.3329/jsr.v3i1.5299 J. Sci. Res. 3 (1), $43-50$ (2011)

\section{Introduction}

Image enhancement is one of the most important issues in low-level image processing. Its purpose is to improve the quality of low contrast images, i.e., to enlarge the intensity difference among objects and background. A lot of methods have been developed and they can mainly be divided into two classes: local and global methods [1,2]. Local methods employ feature-based approach and the local features can be gained by using edge operators or by computing local statistics such as local mean, standard deviation, etc $[1,3]$. They conduct contrast enhancement by modifying the features [4-13]. The common feature-based method is to define the contrast first and enhance image contrast by

\footnotetext{
* Corresponding author: usuf672@yahoo.com
} 
increasing the contrast ratio. Another method uses local histogram modification to enhance image contrast in a local area [14-18], such as: (1) local histogram equalization; (2) local histogram stretching; and (3) nonlinear mapping methods (square, exponential, and logarithmic function).

Histogram equalization is a histogram specification process which consists of generating an output image with a uniform histogram (i.e., uniform distribution). In image processing, the idea of equalizing a histogram is to stretch and/or redistribute the original histogram using the entire range of discrete levels of the image, in a way that an enhancement of image contrast is achieved. Histogram equalization is a technique commonly used for image contrast enhancement, since it is computationally fast and simple to implement.

Low contrast structures need to be resolved in all kinds of digital medical images; e.g, computed tomography (CT), magnetic resonance (MR), digital mammography, ultrasound, angiography and nuclear medicine. Obtaining high contrast in the raw image directly from the imaging device is almost always expensive in examination time or X-ray dose to the patient. In many cases, low contrast can be considered as a result of bad distribution of pixel intensities over the dynamic range of the display device. This suggests the application of contrast enhancement methods in an attempt to modify the intensity distribution of the image. Global methods are mainly implemented by using histogram modification approaches. One of the most commonly used methods is histogram equalization. The main idea of histogram equalization-based methods is to reassign the intensity values of pixels to make the intensity distribution uniform to utmost extent $[16,19,20]$.

The proposed global histogram equalization method is very effective not only in enhancing the entire image but also in enhancing the textural details. It also makes the change of the order of gray levels of the original image completely controllable. Therefore, it can enhance the contrast of the images more effectively. For the data acquisition in this paper, the micro-focus X-ray source (L8121-01, Hamamatsu, Japan) and a commercially available flat-panel detector (C7943CP-02, Hamamatsu, Japan) was used as a $2 \mathrm{D}$ digital $\mathrm{x}$-ray imager in the micro-CT system. The flat-panel detector consists of a $1248 \times 1248$ active matrix of transistors and photodiodes with a pixel pitch of $100 \mu \mathrm{m}$, and a CsI:TI scintillator. The axis-of-rotation was positioned on the line connecting the x-ray focal spot and the center of the detector plane. A micro-CT image of size 512x512 and 16bit signed integer was used as experimental data in this paper.

This paper is organized as follows: Section 2 describes the mathematical description of the proposed algorithm; Section 3 presents the experimental result of contrast enhancing of medical image and finally conclusions are pointed out in section 4 .

\section{Mathematical Description}

The procedure adopted for proposed histogram equalization method is as follows: 
(1) Input image is level offset by the minimum grey level value to make the minimum grey level value to be 0 .

(2) The probability of each grey level is calculated from the available ROI grey levels.

(3) Cumulative distribution function of all the available grey levels in the ROI is calculated using the following equation:

$$
\sum_{j=0}^{k} \frac{n_{j}}{n} \quad k=0,1,2,3, \ldots \ldots \ldots, L-1
$$

where $L$ is the maximum grey level possible (ex. 65535 for the given 16-bit image) in the image, $n j$ / $n$ is the probability of the $j$ th grey level present in the ROI.

(4) Update each pixel in ROI using the following equation:

$$
s_{k}=T\left(r_{k}\right)=(L-1) \sum_{j=0}^{k} \frac{n_{j}}{n} \quad k=0,1,2,3, \ldots \ldots \ldots . ., L-1
$$

where, $s_{k}$ refers to the updated pixel and $r_{k}$ refers to the original pixel.

We also enhance the contrast of the image using local statistics [16-18] to compare the result with the image enhancement using global histogram equalization. The procedure that we follow to enhance the given image using local statistics is as follows:

(1) Take a mask of $3 \times 3$ and slide it over each pixel one by one, from left to right and top to bottom. We chose local area $(3 \times 3)$ for image enhancement. Because the size of the local area should be as small as possible in order to preserve detail.

(2) Check for any background pixel available in the mask and if available avoid that region for processing.

(3) At each slide of mask, calculate mean and standard deviation of the $3 \times 3$ region of the image.

(4) Compare the values of local mean and standard deviation with the global mean and standard deviation and select the center pixel $f(x, y)$ of mask for processing according to the following logic:-

$$
g(x, y)=\left\{\begin{array}{cc}
E . f(x, y) & \text { if } m_{s_{x y}} \leq k_{0} m_{G} \text { and } k_{1} \sigma_{G} \leq \sigma_{s_{x y}} \leq k_{2} \sigma_{G} \\
f(x, y) & \text { Otherwise }
\end{array}\right.
$$

where $E, k_{0}, k_{1}, k_{2}$ are specified parameters; $m_{G}$ is the global mean of the input image; and $\sigma_{G}$ is its global standard deviation.

\section{Experimental Result and Discussions}

We now present some experimental results obtained by applying the proposed method to the enhancement of gray level micro-CT (micro computed tomography) medical images. 


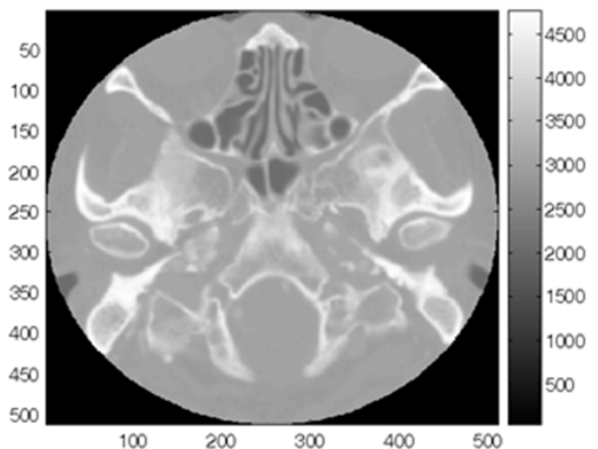

(a)

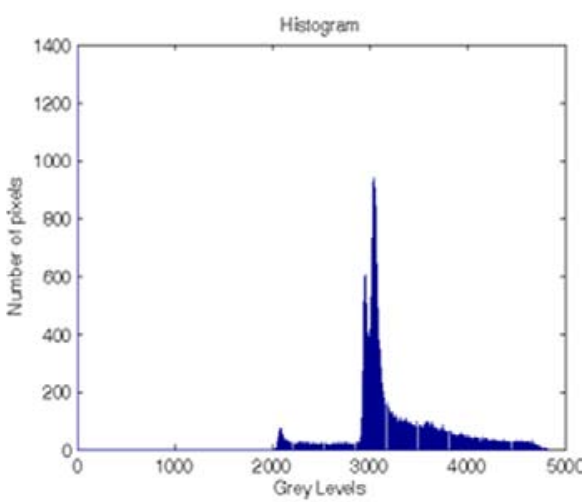

(b)

Fig. 1. (a) Original CT image without histogram equalization (b) Histogram of CT image.

Fig. 1 shows the input CT image to be histogram equalized and its corresponding histogram. Fig. 1(a) shows the original CT image of size $512 \times 512$ and 16-bit signed integer. The given CT image was level offset by the minimum value of -3024 to make the minimum value as 0 . As can be seen from the histogram, there are more grey levels in the range 2000 to 4000 and the full dynamic range of 0 to 65535 is not covered. Moreover, even in the given range there are more pixels at around 3000 grey level. Thus, histogram equalization might be a proper way to enhance the contrast of the given image.

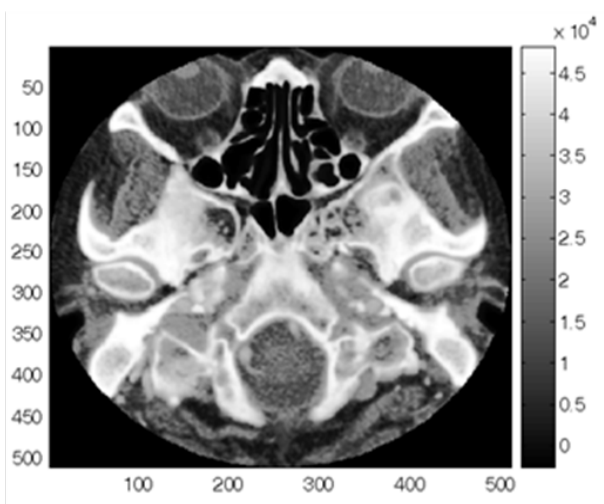

(a)

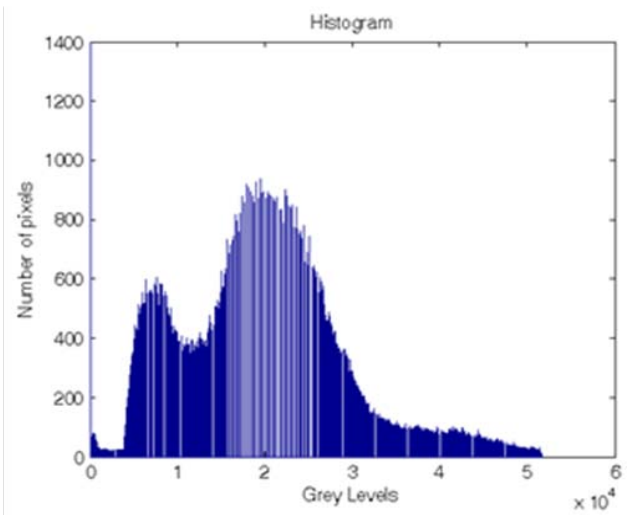

(b)

Fig. 2. (a) Histogram equalized CT image, (b) Histogram of image in (a).

The results of histogram equalization are shown in Fig. 2. Fig. 2(a) shows the histogram equalized image and (b) shows the histogram of the equalized image. Comparing Fig. 1(a) and Fig. 2(a) there is a huge contrast enhancement in the boundary regions of the CT image. The regions which were embedded in the boundary regions of 
Fig. 1(a) can be clearly seen in the Fig. 2(a). Comparing Fig. 1(b) and Fig. 2(b), the histogram of Fig. 2(b) shows a spread in the distribution of grey levels from range 0 to 5000. Since, there is a spread in the grey level distribution over almost complete dynamic range there is a huge contrast improvement.

Now we enhance the contrast of the input image of Fig. 1 (a) using local statistics of mean and standard deviation. The global mean of image refers to the average intensity of image and global standard deviation infers the overall contrast of image. Thus, comparing the global mean and standard deviation values with the local mean and standard deviation in some chosen region, image might be enhanced using some criterion.

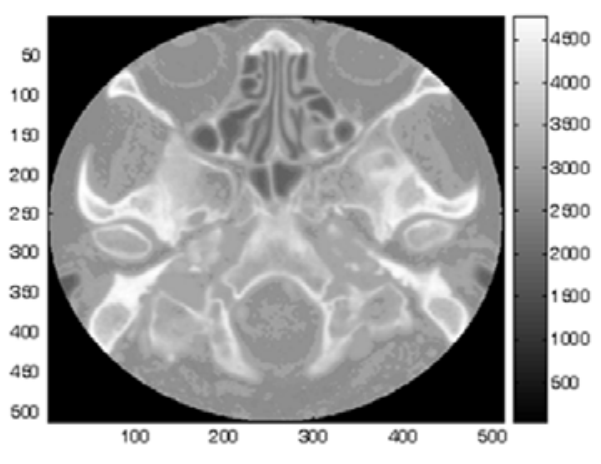

(a)

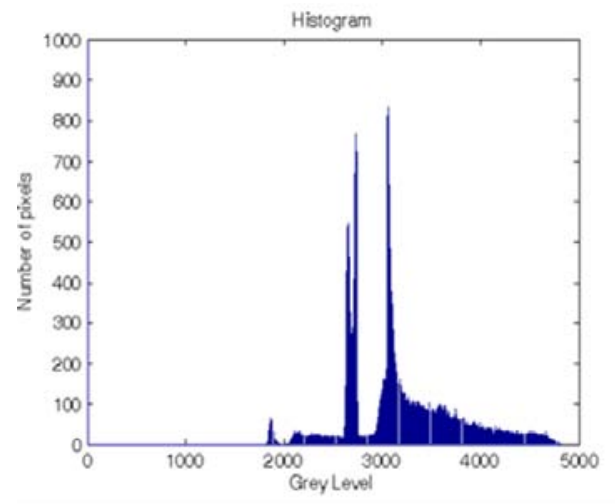

(b)

Fig. 3. (a) CT image after local processing (b) Histogram of CT image after local processing.

The result of the above procedure for enhancement using local statistics is shown in Fig. 3. Fig. 3(a) shows the processed image based on local statistics and Fig. 3(b) shows the histogram of image in (a). The values of the parameters in Eq. (3), obtained using trial and error, are shown in Table 1.

Table 1. Parameter values.

\begin{tabular}{llll}
\hline$E$ & $k_{0}$ & $K_{1}$ & $K_{2}$ \\
\hline 0.9 & 1.2 & 0.001 & 0.1 \\
\hline
\end{tabular}

Comparing Fig. 1(a) and Fig. 3(a), some of the hidden regions in low contrast areas of image can be seen in Fig 3 (a). However, there is not much enhancement in contrast. Comparing the histograms in Fig. 1(b) and Fig. 3(b), the histogram in Fig. 3(b) has exactly same dynamic range spreading except that the distribution of few pixels have increased, ex) at around 2500 grey level. However, the enhanced image still seems to be a low contrast image, since the full dynamic range is not covered by histogram. 
Comparing the histograms obtained from both the enhancement techniques (Fig. 4), we can infer that the histogram after histogram equalization has spread of grey levels over almost complete dynamic range from 0 to 50,000 grey level values. This indicates that enhanced image using this technique would show an image with high contrast which can be seen in Fig. 5(a). However, the histogram in Fig. 4(b) corresponding to the enhancement technique using local statistics does not show any spread of grey level distribution over the complete dynamic range, except some amplification of distribution at certain grey levels ex) at around 2500. Thus, a better contrast image cannot be expected as can be seen in Fig. 5(b).

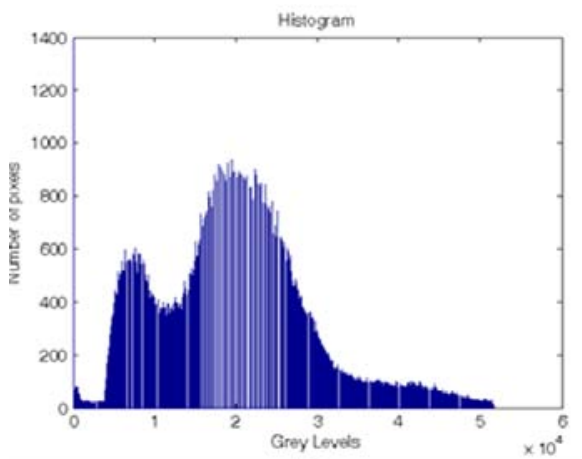

(a)

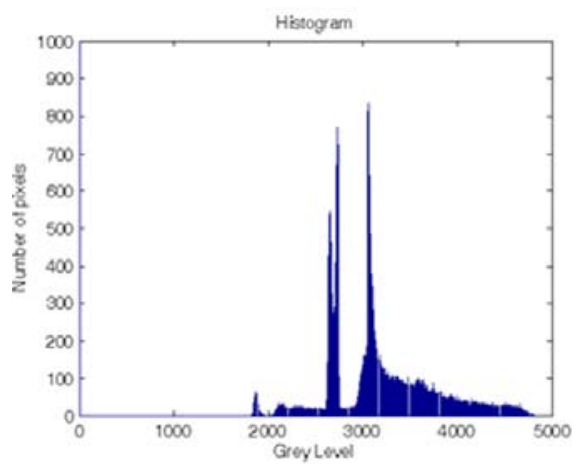

(b)

Fig. 4. Histogram of CT image (a) after histogram equalization, and (b) after enhancement using local statistics.

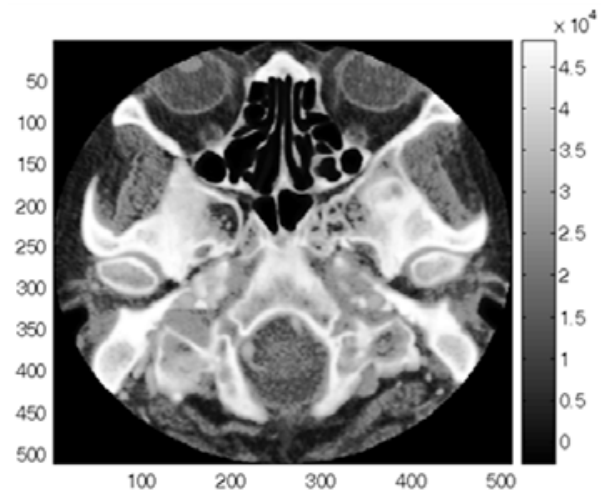

(a)

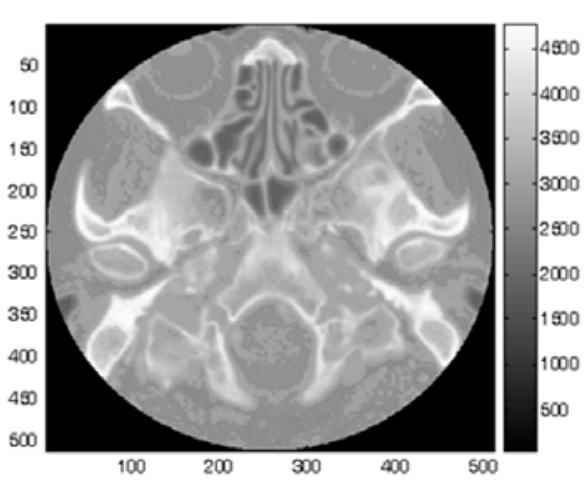

(b)

Fig. 5. CT image (a) after histogram equalization, and (b) after enhancement using local statistics.

The image enhancement method using local statistics cannot enhance the input image properly and the global histogram equalization seems to be a better approach for the input CT image. 
Histogram equalization is simple and effective in enhancing the low contrast image only if (a) it contains single object or (b) no apparent contrast change between object and background. Since the above conditions cannot be always met, the global methods have both over-enhancement and under-enhancement problems.

\section{Conclusion}

Image enhancement is one of the most important issues in low-level image processing. All the methods are based either on local information or on global information. A novel approach using global information to enhance image is studied in this paper. Experimental results show that it is very effective in enhancing images with low contrast, regardless of their brightness. The proposed algorithm is very efficient not only in enhancing the entire image but also in enhancing the textural details. Our global histogram equalization-based method is better at preserving the brightness of the image compared to the others local statistics based method because the intensity values of pixels are re-assigned to make the intensity distribution uniform to utmost extent.

\section{References}

1. A. K. Jain, Fundamentals of Digital Image Processing (Prentice-Hall, 1989) ISBN 0-13336165-9.

2. J. Y. Kim, L. S. Kim, S. H. Hwang, IEEE Transactions on Circuits and Systems for Video Technology 11 (4), 475 (2001). doi:10.1109/76.915354

3. W. K. Pratt, Digital Image Processing, $3^{\text {rd }}$ Ed. (John Wiley \& Sons, 2001) ISBN 0-471-374075. doi:10.1002/0471221325

4. Z. Chen, Y. Tao, and X. Chen, Appl. Opt. 40 (8), 1195 (2001). doi:10.1364/AO.40.001195

5. B. Smolka, K. W. Wojciechowski, Signal Process 81 (3), 465 (2001). doi:10.1016/S0165-1684(00)00226-7

6. G. Braun and M. D. Fairchild, J. Electron. Imag. 8 (4), 380 (1999).

7. S. C. Matz, R. J. P. de Figueiredo, IEEE International Conference on Image Processing (ICIP '99) 3, 484 (1999).

8. T. Villalobos, I. R, C. Mandujano, and J. A, J. Electron. Imag. 7 (3), 641 (1998).

9. C. Munteanu and A. Rosa, IEEE Transactions on Systems, Man and Cybernetics, Part B 34, 1292 (2004).

10. A. S. Georghiades, P. N. Belhumeur, and D. J. Kriegman, IEEE Int. Conf. on Automatic Face and Gesture Recognition (2000) pp 277-284.

11. G. Ramponi, Signal Process 67 (2), 211 (1998). doi:10.1016/S0165-1684(98)00038-3

12. D. Mukherjee and B. N. Chatterji, Graph. Models Image Process 57, 254 (1995). doi:10.1006/gmip.1995.1024

13. F. Neycenssac, Graph. Models Image Process 55, 447 (1993). doi:10.1006/gmip.1993.1034

14. J. A. Stark, IEEE Trans. Image Process. 9 (5), 889 (2000). doi:10.1109/83.841534

15. H. C. Zhu, H.Y. Francis, and F. K. Lam, Comp. Vision Image Understand. 73 (2), 281 (1999). doi:10.1006/cviu.1998.0723

16. S. D. Chen and A. R. Ramli, Digital Signal Processing 12 (5), 413 (2004). doi:10.1016/j.dsp.2004.04.001

17. S. D. Chen and A. R. Ramli, IEEE Trans. Comp. Electr. 49 (4), 1301 (2003). doi:10.1109/TCE.2003.1261233 
18. S. M. Pizer, E. P. Amburn, J. D. Austin, R. Cromartie, and A. Geselowitz, Comp. Vis. Graph. Image Process 39, 355 (1987). doi:10.1016/S0734-189X(87)80186-X

19. Y. Wang and Q. Chen, B. Zhang, IEEE Trans. Consum. Electron. 45 (1), 68 (1999). doi:10.1109/30.754419

20. Y. T. Kim, IEEE Trans. Consum. Electron. 43, 1 (1997). doi:10.1109/30.580378 